Théologiques

Théologiques

\title{
Le soi, la personne, le « je " Perspectives hébraïques. Mise en dialogue avec un modèle mécanique d'auto-organisations mémorisées
} Post-scriptum — Dialogue

\section{Henri Atlan et Paul Dumouchel}

Volume 12, numéro 1-2, 2004

Le Soi dans tous ses états

URI : https://id.erudit.org/iderudit/011556ar

DOI : https://doi.org/10.7202/011556ar

Aller au sommaire du numéro

Éditeur(s)

Faculté de théologie et de sciences des religions, Université de Montréal

ISSN

1188-7109 (imprimé)

1492-1413 (numérique)

Découvrir la revue

Citer cet article

Atlan, H. \& Dumouchel, P. (2004). Le soi, la personne, le « je " Perspectives hébraïques. Mise en dialogue avec un modèle mécanique d'auto-organisations mémorisées : post-scriptum — Dialogue. Théologiques, 12(1-2), 71-93.

https://doi.org/10.7202/011556ar

Tous droits réservés ( Faculté de théologie et de sciences des religions, Université de Montréal, 2005
Ce document est protégé par la loi sur le droit d'auteur. L'utilisation des services d’Érudit (y compris la reproduction) est assujettie à sa politique d'utilisation que vous pouvez consulter en ligne.

https://apropos.erudit.org/fr/usagers/politique-dutilisation/ 


\title{
Le soi, la personne, le « je» \\ Perspectives hébraïques. Mise en dialogue avec un modèle mécanique d'auto-organisations mémorisées
}

\author{
Henri AtLan \\ Professeur émérite de biophysique \\ Universités de Paris VI et de Jérusalem \\ Dircteur d'études à l'EHESS, Paris
}

\section{Introduction: soi inconscient et conscience du soi}

Existe-t-il un soi inconscient? Oui, bien sûr, le soi biologique. Car le soi, comme la conscience, n'est pas une réalité qui existerait en tout ou rien. Certaines choses ou certains êtres auraient un soi et d'autres pas. Il existe différentes sortes, ou différents degrés, de soi, comme de conscience, et, de ce point de vue, le titre de ce numéro, Le Soi dans tous ses états, me semble tout à fait adéquat. Comme à propos de la conscience, se pose alors la question de l'intégration éventuelle de ses différents aspects et des propriétés qui leur sont afférentes. Et comme pour la conscience, cette intégration n'est pas qu'une simple addition: ses différentes composantes se modifient les unes les autres et leur ensemble est différent de la seule addition de leurs propriétés, prises chacune isolément. Le soi inconscient — ou plutôt les différents soi inconscients — sont modifiés par l'existence du soi conscient, et bien évidemment celui-ci ne peut pas être considéré isolément, désincarné en quelque sorte, sans rapports avec le corps inconscient qu'il est aussi.

Une des expressions les plus spectaculaires du soi biologique est la distinction qu'effectue le système immunitaire entre le soi et le non-soi à travers des structures moléculaires et des activités des cellules de notre corps. On peut aussi concevoir que l'activité inconsciente de notre cerveau produit et exprime un soi inconscient.

Aussi bien le soi immunologique que le soi cérébral inconscient ne sont pas donnés une fois pour toutes: ils se constituent à travers les activités du système immunitaire et du système nerveux - d'ailleurs en relation l'un avec l'autre et avec le reste de l'organisme, notamment le 
système endocrinien dont on a de plus en plus de mal à les différencier. Pour l'un comme pour l'autre, le stockage en mémoire des évènements de leur histoire, de leur développement et des rencontres en partie aléatoires avec d'autres individus, d'autres soi et d'autres éléments de leur environnement, est un élément déterminant de la structure et de l'évolution du soi. Ce rôle de la mémoire et de l'histoire de l'individu dans la constitution du soi, nous le retrouvons aussi quand nous essayons de cerner la nature du soi conscient. De quoi avons-nous conscience quand nous avons conscience de nous-mêmes? Autrement dit, en quoi le soi conscient est-il différent de l'inconscient? On peut répondre: en ce que nous en avons conscience! Mais de quoi avons-nous conscience, puisqu'il est clair que nous n'avons pas conscience de l'activité des lymphocytes qui constituent notre système immunitaire, ni de l'activité des neurones de notre cerveau? Il semble que pour concevoir ce dont nous avons ainsi conscience, nous devrions faire le détour par la question de la conscience. Qu'est-ce que la conscience en relation avec l'activité des cellules de notre corps? C'est le problème corps-esprit classique, que l'on peut difficilement éviter: émergence de la conscience et des activités de l'esprit d'un individu, en relation avec le développement de son corps et notamment de son cerveau.

Je ne vais pas tenter de résoudre ce problème ici, évidemment. Mais nous l'approcherons par la bande, si l'on peut dire, sachant que la position qui me semble la plus féconde, bien que peut-être pas la plus facile, est celle d'un monisme spinoziste, ni idéaliste ni matérialiste, affirmant de façon radicale une unité psychophysique. Mais nous devons faire encore un autre détour, cette fois par la question de la personne. Et cette question n'est pas qu'individuelle, elle est aussi de nature sociale. Car le soi dont nous avons conscience, nous en parlons; verbalement ou non verbalement, il s'exprime. C'est le soi qui dit «je » en parlant de lui ou d'ellemême. Ce soi se constitue dans nos rapports avec autrui, c'est-à-dire avec d'autres êtres parlant, c'est-à-dire humains, avec qui nous vivons en société, soit directement, soit indirectement, à travers le ou les groupes sociaux dans lesquels nous sommes nés, avons grandi et sommes devenus nous-mêmes des êtres humains doués de parole. C'est pourquoi, nous verrons que la façon peut-être la plus adéquate de rechercher le soi conscient, lequel semble s'évanouir quand on croit avoir mis la main dessus, est de réfléchir à la nature, elle aussi très présente et fuyante à la fois, de ce qui s'exprime quand quelqu'un parle et dit «je». On peut éventuelle- 
ment se dire «je" à soi-même. Cette personne prend alors la place d'un toi dans un dialogue intérieur, tout en assumant celle de son moi. En effet, le «je» ne doit pas être confondu avec le moi ou l'ego, car ceci désigne une réalité consciente certes, mais peut-être trop consciente et pour cela enfermée ou chosifiée dans une image de soi bien déterminée, objectivée si l'on peut dire, d'où le sujet de la parole, qui dit "je», a disparu. La première personne du singulier qui dit «je» est tout à la fois un moi, un toi et un soi, et en disant «je ", elle assume ces trois personnes tout en restant une, la première.

Avant d'en arriver là, voyons donc ce que l'on peut dire, très schématiquement, sur cette question, elle aussi source de controverses: qu'est-ce qu'une personne?

\section{La personne humaine}

\subsection{La notion de personne absente des sciences de la nature}

Pendant longtemps, il a été entendu que les attributs de la personne étaient liés à des propriétés intrinsèques de l'individu humain et que le rôle des sciences de la nature, et de la philosophie, était de découvrir et de circonscrire ces propriétés. Tout naturellement, des philosophes comme Emmanuel Kant et ses successeurs prenaient appui sur la biologie vitaliste de leur temps pour faire coexister les sciences de la nature, physicochimiques et mécaniques, avec l'étude rationnelle d'une morale et d'un droit fondés sur l'observation de finalités dans la nature. Une belle construction métaphysique a cru pouvoir concilier le mécanicisme physicochimique et une morale rationnelle fondée sur le libre arbitre et la dignité de la personne humaine en tant que fait naturel. Ceci a permis à l'humanisme occidental d'exister pendant ces deux derniers siècles.

Mais cette conciliation ne pouvait tenir que tant que les théories finalistes et vitalistes en biologie gardaient encore un caractère vraisemblable. Nous devons constater aujourd'hui que le succès en biologie du mécanicisme physicochimique a eu comme corollaire la disparition progressive de la notion de personne - et donc celles de liberté, de responsabilité, etc. - de l'ensemble des représentations scientifiques que nous pouvons nous faire de la nature.

L'ambition de la biologie moderne est de rendre compte des comportements animaux, et aussi des nôtres, non pas en invoquant notre âme et 
notre liberté, mais à l'aide de déterminismes d'interactions physicochimiques entre les molécules qui constituent notre corps. Chaque fois que cette ambition se réalise, elle exclut d'autant la place que l'on peut accorder à la liberté d'une personne, entendue au sens kantien d'un domaine suprasensible de la liberté, qui s'exprimerait dans notre libre arbitre et nos choix libres éclairés par la raison. Et pourtant, la vie sociale ne peut pas se passer du droit, qui semble impliquer d'une façon ou d'une autre, du fait de cette tradition philosophique dominante, la réalité de ce libre arbitre.

Il y a donc là une opposition et c'est là tout l'intérêt d'un retour à Spinoza et à l'idée d'une liberté différente du libre arbitre, laquelle posait déjà les conditions d'une éthique, impliquant, entre autres, respect des personnes et respect par les personnes des lois de la société, alors même que l'idée d'un déterminisme mécanique universel était renouvelée par rapport à celle des anciens stoïciens et de certains courants de la tradition juive. Cette nécessité d'une éthique de la liberté par la connaissance des déterminismes s'imposait à Spinoza, à la naissance de la science moderne au XVII ${ }^{e}$ siècle, alors que la volonté libre ne pouvait être pour lui qu'une illusion produite par l'ignorance des causes des affections du corps.

L'opposition entre une science du vivant de plus en plus dépersonnalisée et les nécessités de l'éthique et du droit toujours fondées sur la réalité de la personne, pose des problèmes concrets qui appellent des décisions juridiques et administratives. Confrontés à ces problèmes, il faut donc d'abord renoncer à l'idée de définir la personne humaine comme une réalité dans l'ordre des faits, si ces faits ne doivent être instruits que par les sciences de la nature - biologie et psychologie comprises - et qu'au contraire, il faut accepter l'idée qu'il s'agit là d'une réalité de droit.

En effet, que peut nous dire aujourd'hui la biologie sur la personne? En fait, pas grand-chose, bien qu'elle nous en dise beaucoup sur l'individu et sur le processus d'individualisation. Mais ce n'est pas la même chose. On sait aujourd'hui que l'individualité biologique dans l'espèce humaine est poussée très loin, comme c'est évident, par exemple, de par le caractère unique du système immunitaire de chaque individu après quelques années de développement. C'est cela qui explique que les greffes de tissu d'un individu humain sur un autre sont normalement rejetées. Mais même cette individualité-là n'est pas donnée d'emblée. Elle est le résultat de la maturation du système immunitaire qui commence dans l'embryon et se poursuit dans les premières années de la vie. 
Le seul caractère véritablement unique propre à chaque individu dès la fécondation, c'est sa carte génétique, résultat de ce qu'on appelle la loterie génétique de la reproduction sexuée. Mais cela n'est pas propre à l'espèce humaine et ne peut donc pas servir de support à une éventuelle définition biologique de la personne humaine.

En outre, l'unicité du génome ne suffit pas pour déterminer l'unicité biologique d'un individu, du fait des mécanismes épigénétiques qui contribuent à sa maturation et à son développement, comme on vient de le voir à propos du système immunitaire. Le développement du cerveau s'effectue aussi de cette façon. C'est pourquoi deux individus génétiquement identiques, comme deux vrais jumeaux ou deux individus clonés, sont déjà biologiquement différents.

\subsection{La notion occidentale de personne enracinée dans le droit romain}

En fait, à l'origine, l'idée de personne semble être issue non pas de la réflexion scientifique, ni même philosophique, mais du droit et essentiellement du droit romain. La première idée est que les hommes sont considérés comme personnes en tant qu'êtres dont le statut juridique est posé comme différent de celui des choses et des animaux. En particulier, leur sont attribués une responsabilité, des obligations légales et des droits, et surtout une dignité dont le respect est au cœur des principes sur lesquels se fondent les droits de l'homme en Occident. En même temps, la personne humaine ne saurait exister comme abstraction pure sans support physique, et celui-ci est évidemment le corps humain; d'où le deuxième principe, juridique aussi: après la distinction des personnes et des choses, celui d'indivisibilité du corps et de l'esprit.

Ces idées, distinction entre personne et chose et indivisibilité du corps et de l'esprit, sont fortement déterminées par le contexte culturel et linguistique où elles se sont développées, à savoir, la civilisation occidentale chrétienne, certes héritière du monde judéo-grec, mais aussi de Rome, surtout en ce qui concerne son droit. Il est évident que la notion de personne humaine sera diversement appréciée dans d'autres civilisations, par exemple dans celles où la réincarnation joue un rôle important, ou là où un animal comme une vache a un caractère au moins aussi sacré que l'homme, ou encore, de façon générale, dans un contexte culturel où la notion d'âme ou d'esprit n'est pas spécifique de l'homme et peut être étendue aux animaux, aux plantes ou à tout autre chose. 


\subsection{La notion hébraïque de personne}

Voyons ce qu'il en est de la notion de personne dans la civilisation hébraïque et dans le droit talmudique qui en est issu. Ce droit est différent du droit romain sur plus d'un point.

\subsubsection{Terminologie hébraïque et mise en récit de Gn 1-2}

Tout d'abord, il est remarquable qu'on ne trouve pas en hébreu d'équivalent du "persona» latin, c'est-à-dire de mot qui traduise exactement la notion de "personne humaine». Suivant le contexte, on trouve adam ou énoch («homme» en général), néfèch (terme qui désigne un aspect de l'âme), gouf («corps»), ich (un autre mot pour «homme»), ou encore panim ( «isage»). Il serait facile de montrer qu'aucun de ces termes n'a la signification exacte du mot "personne». Ainsi, néfèch, aussi traduit par "âme ", désigne l'animation d'un corps vivant et, en ce sens, se rapproche de l'idée de personne, où se trouvent réunis de façon indivise le corps et l'esprit. Cependant, l'expression néfèch 'hayia, qui, appliquée à l'homme, peut être traduite par "personne vivante », est employée dans le récit biblique indifféremment pour l'homme et l'animal (voir Gn $1,20.21 .24 ; 2,7)$.

Le terme qui se rapprocherait le plus de l'idée latine de «personne » serait peut-être un dérivé du substantif $i c h$, à savoir le qualificatif $i c h i$, adjectif qui a acquis en hébreu moderne le sens de "personnel », tandis que les substantifs $i c h$ et icha veulent simplement dire, respectivement, «homme» et «femme». Un substantif dérivé de la même racine, ichiout, s'en rapproche un peu, mais il désigne en fait la personnalité de quelqu'un plutôt que la notion de personne. Quant à un autre substantif, ichout, toujours dérivé de la même racine, il désigne l'ensemble du domaine des relations entre homme et femme, c'est-à-dire en gros la sexualité.

Ainsi, il existe bien apparemment un rapport entre la racine hébraïque $i c h$ ou icha et la notion romaine de personne, mais ce rapport ne s'exprimerait - à la rigueur - que dans le qualificatif ichi ("personnel»), et dans aucun substantif. Pour entrevoir la nature possible de ce rapport, le récit biblique peut nous aider. Relevons en particulier la façon curieuse dont le récit de la séparation en homme et femme de l'adam androgyne de la Genèse fait apparaître pour la première fois le mot $i c h$. 
Après que la femme eut été séparée, Adam la vit, la rencontra, et l'appela $i c h a$, féminin de $i c h$, car, dit le texte, elle fut prise de ich (Gn 2,23). Mais le récit est énigmatique, car elle avait été "prise» d'Adam. Celui-ci avait nommé les animaux et nomma aussi la femme, mais il se nomma luimême ich par la même occasion, car on n'avait jamais rencontré dans le récit biblique, jusque là, de ich d'où $i c h a$ aurait pu être prise. À partir de là, seul le mot icha sera utilisé pour désigner une femme, tandis que les deux mots adam et $i c h$ vont continuer d'être utilisés suivant le contexte pour désigner un homme. De cela nous pouvons tirer plusieurs leçons.

On nous décrit d'abord une transformation de adam en ich: adam, masculin de adama, la terre dont il est issu, est transformé en ich, masculin de «femme». Il s'agit plus exactement d'une transformation du couple tellurique adam-adama, c'est-à-dire humain androgyne-terre nourricière, en couple personnalisé, homme-femme. Ensuite, cette transformation apparaît comme un processus, toujours en cours, de personnalisation, ou même d'hominisation, qui serait donc plus accompli et plus précoce pour la partie féminine de adam que pour sa partie masculine. C'est pourquoi aucun substantif ne pourrait désigner ce processus en ce qu'il est toujours en cours; ni ich ni icha, car chacun d'entre eux serait trop statique, trop limité, enfermé dans l'ego d'un homme particulier ou d'une femme particulière.

Autrement dit, la personne humaine serait instituée, non pas dans un individu — c'est pour cette raison qu'il n'y a pas de mot unique pour la désigner - , mais dans la rencontre et la reconnaissance par le regard d'un autre. Et pas de n'importe quel autre: pas l'animal, trop différent, et pas l'autre du même sexe, trop semblable, mais l'autre du sexe différent.

Tout se passe donc comme si l'humanité n'était au départ qu'une propriété collective, celle de l'espèce adamique pour ainsi dire, comme l'animalité d'une autre espèce, et qu'il n'y avait, à ce stade, rien de personnel dans les individus de cette espèce. Pour que cette propriété de personne devienne celle d'individus vivants, et en fasse autre chose que de vivants animaux, il faut que ces individus acquièrent cette humanité au cours de leur développement, en quelque sorte que non seulement ils s'hominisent, mais aussi qu'ils s'bumanisent. Le signe que cette humanisation s'est accomplie dans l'individu, ce qui permet de le désigner, non seulement par le nom générique adam, mais par celui personnalisé de ich ou icha, c'est la possibilité qu'ils ont de se reconnaître dans la rencontre homme-femme. 
Reste évidemment à se poser la question : mais qu'est-ce que l'homme reconnaît dans la femme et qu'est-ce que la femme reconnaît dans l'homme, qui soit différent de ce qu'ils peuvent reconnaître l'un et l'autre chez l'animal? Autrement dit, qu'est-ce qui est donné dans adam, qui lui permet de s'humaniser et d'être reconnu comme ich ? Ou encore, qu'estce qui, dans adam, est potentialité de ich ou icha, ou, comme nous dirions aujourd'hui peut-être, potentialité de personne humaine?

Dans ce contexte, on peut montrer que ce que l'on reconnaît ainsi immédiatement, c'est simplement la forme du corps humain, ce que la Bible appelle le tsélèm, image ou icône, quand elle dit qu' «Adam fut créé mâle et femelle à l'image d'Élohim» (voir Gn 1,27). Je ne peux pas entrer ici dans les détails, mais seulement dire que ceci ne renvoie pas à une certaine image de Dieu que nous connaîtrions par ailleurs. C'est le contraire. C'est cette forme humaine en tant que telle qui est en quelque sorte divinisée, par abstraction et généralisation de sa structure, dans un monde où les divinités sont multiples, comme l'indique le pluriel du nom «Élohim».

\subsubsection{Applications éthiques et juridiques}

Qu'on ne croit pas, malgré les apparences, qu'il ne s'agisse là que de métaphores poétiques ou de mythes qui n'auraient pour objet que l'édification religieuse. Il s'agit en fait de considérations qui ont des conséquences éthiques et juridiques immédiates, concernant directement les problèmes pratiques de définitions et de limites, associés à la notion de personne humaine.

Une façon radicale et actuelle de poser ces problèmes consiste à se demander qui est «l'homme", sujet des droits de l'homme. À quoi le reconnaît-on? N'est-ce pas, en fin de compte, à la forme de son corps? Le signe le plus visible, le plus évident, de l'humanité d'un individu est son corps. Le corps d'homo sapiens, avec son visage, est tout de suite perçu comme tel. Sans investigation savante, il n'est confondu avec celui d'aucune autre espèce ni avec celui d'un robot, même intelligent. L'humanité de l'espèce, c'est-à-dire de tous, est concentrée dans le corps de chacun, dans la réalité de sa physiologie et dans l'apparence de sa forme où s'exprime aussi la réalité de son existence sociale. Cette définition empirique de l'humanité peut servir de principe régulateur pour résoudre les difficiles problèmes éthiques que posent les interventions biomédicales 
en début et en fin de vie. Par exemple, la fameuse question: à partir de quand un embryon est-il une personne? Les critères biologiques, quelqu'ils soient - fécondation, différenciation du blastocyte à cinq jours, apparition des ébauches nerveuses à quatorze jours, etc. - , sont toujours arbitraires même s'ils peuvent avoir quelque utilité.

Plutôt que de chercher un critère de fait, d'origine scientifique, toujours fuyant et de toute façon contestable, autant accepter un critère de droit fondé sur notre sensibilité et notre perception immédiate. Autrement dit, ce sera notre capacité de percevoir la forme du corps humain et en particulier de son visage - c'est-à-dire notre propre forme - dans un individu vivant qui sera le critère par lequel nous y reconnaîtrons une personne; non pas donc au terme d'une longue analyse scientifique et philosophique, mais immédiatement, par projection et identification à partir de notre propre forme.

L'idée de critère de reconnaissance d'une personne sur la base de la perception immédiate et extérieure de la forme, du tsélèm, par projection et identification, donne un sens à des considérations apparemment bizarres du Talmud et de Maimonide, sur l'avortement et le statut de l'embryon avant quarante et un jours.

On sait que le droit talmudique considère quarante et un jours comme un seuil en-deçà duquel un avortement n'est pas un avortement, car ce qui est expulsé n'est pas reconnu comme un enfant. La raison qui en est donnée est que "tout ce qui n'a pas de forme humaine n'est pas un enfant" (Talmud de Babylone, Nidda, 21a). Autrement dit, un individu n'est considéré comme un enfant, avec un statut différent d'une chose ou d'un animal, que si nous y percevons le tsélèm, c'est-à-dire la forme humaine et notamment son visage. Et Maimonide détaille dans son traité les caractéristiques proprement humaines du visage. C'est ainsi que tant que les yeux sont sur les côtés de la tête, il s'agit encore d'une forme animale qui ressemble, comme il le dit, à une mouche. Il faut attendre qu'ils se soient rapprochés et soient situés sur la face, pour qu'on y perçoive un visage humain. D'où, entre autres, ce délai de quarante et un jours.

Pour nous, aujourd'hui, même si ce seuil peut être modifié, en plus ou en moins suivant les problèmes considérés, le principe qui implique une attitude pragmatiste, non substantialiste, peut rester le même. Autrement dit, c'est notre reconnaissance d'une forme et d'un visage humains qui nous fait attribuer le statut d'une personne, et non la recherche de 
propriétés intrinsèques qui témoigneraient d'une nature de personne humaine. Cette position a le grand avantage de fournir un critère assez efficient pour résoudre les problèmes de limite. En ce qui concerne l'embryon, cela nous conduit à accepter l'idée, en quelque sorte, d'un préembryon, où il ne s'agirait que d'un amas de cellules sans forme et où notre sensibilité immédiate, qui n’y voit pas de personne humaine, pourrait être assumée - en ce qui concerne, évidemment, son présent, à l'exclusion de toute finalité de fabrication concernant une personne future qui résulterait éventuellement de son développement.

Ce critère est aussi efficient en ce qui a trait, par exemple, au respect dû au cadavre. C'est la forme humaine encore présente, à laquelle est attribuée ici une dignité de personne.

En outre, ce même critère s'étend très facilement au problème qui se pose maintenant de plus en plus, de l'expérimentation animale. Là aussi, quand on est obligé d'expérimenter sur l'animal, notre sensibilité est déterminée par notre identification plus ou moins grande avec les différents objets d'expérimentation possibles. Le biologiste considère qu'il est bien plus «humain» de travailler sur des cellules en culture, même si elles sont d'origine humaine, que de travailler sur des animaux. Pourquoi? Parce que ces animaux ressemblent extérieurement — du point de vue de notre perception immédiate et non savante - , bien plus aux hommes que des cellules. Il s'agit là d'une identification relative du chercheur humain avec son objet d'expérimentation. Le problème est d'autant plus difficile que l'expérimentation comporte des risques graves, puisque, finalement, ces objets d'expérience - cellules ou animaux — sont très souvent détruits, ou — dans le cas des animaux — «sacrifiés », comme on dit dans la littérature spécialisée. Encore une fois, cette identification n'a pas grand-chose de scientifique: c'est une identification immédiate de sympathie. On s'identifie plus facilement avec un singe qu'avec une souris, et plus avec une souris qu'avec une grenouille, et plus avec une grenouille qu'avec une mouche, et plus avec une mouche qu'avec des cellules en culture, même si ces cellules sont d'origine humaine.

Certains veulent trouver dans l'existence d'une conscience un critère de distinction, qui permettrait, par exemple, de différencier les grands singes des autres animaux. Mais ce critère est tout aussi discutable que les autres en ce que l'apparition de la conscience est un phénomène progressif, dans la phylogenèse comme dans l'ontogenèse. En fait, c'est le sens de notre sensibilité immédiate qu'il s'agit finalement de retrouver et 
d'assumer, plutôt que de chercher une illusoire définition essentialiste de la nature de la personne humaine, qu'on la dise potentielle ou réelle.

\subsection{Conclusion provisoire: pour un «dialogue» entre sciences et éthique}

On voit que la vérité scientifique sur la nature de la nature n'est pas toujours nécessairement la plus pertinente pour les questions de droit et de personnes. Cela ne veut pas dire, inversement, que cette vérité scientifique puisse être ignorée ou négligée. Il ne s'agit donc pas, sous prétexte de défendre la morale, d'accepter un retour aux confusions du Moyen Âge et à un obscurantisme, même paré des vertus de la religion. Il ne s'agit pas d'ignorer les mérites des sciences et des techniques sous prétexte qu'elles n'ont rien à nous dire sur le "comment vivre». M. de la Palisse dirait sans doute qu'on ne peut pas poser les problèmes sans savoir comment ils se posent en vérité. C'est donc à un va-et-vient sans cesse renouvelé que nous devons nous attacher entre recherche objective des vérités scientifiques sur les réalités de la nature, d'un côté, et souci de justice et de dignité, de l'autre, même si c'est bien plus difficile aujourd'hui qu'autrefois, puisque l'illusion d'une unité toute faite entre vérité et justice, entre le vrai et le bien, a été dissipée.

\section{Le «je»}

Nous n'avons envisagé jusqu'ici que la personne ou le tsélèm dans sa généralité. Qu'en est-il de son individualité, de l'unicité de chaque personne, sa singularité?

\subsection{La notion $d u$ "je» illustrée dans la symbolique du dieu des personnes}

La même tentation existe d'en rechercher une définition objective. Et c'est encore vers la génétique que l'on se tourne pour chercher la signature concrète de cette unicité. Mais il y a là un danger certain, car l'individualité génétique, si elle est indiscutable, n'est pourtant pas, comme on l'a vu, le propre de l'humain. L'individualité génétique n'est donc pas forcément le support d'une personne humaine: elle implique la possibilité d'individualités impersonnelles. L'usage des empreintes génétiques ne doit pas induire en erreur. Il s'agit précisément d'empreintes, c'est-à-dire de traces, qui, comme les empreintes digitales, signent une individualité sans la déterminer, ni même en être le support. 
Ici aussi, il vaut donc mieux chercher ailleurs que dans la science objective, par exemple dans la grammaire et dans la symbolique du récit mythique, ce qui fait la réalité de chaque personne humaine et sa singularité, ce que l'on peut considérer comme son soi, en tant qu'on en est conscient et qu'on le désigne en disant «je».

Une telle symbolique nous est suggérée par l'idée du «dieu des personnes ", qu'il faut comprendre, non pas comme celle du Dieu personnel, qui serait lui-même une personne, en une ou en trois, mais plutôt l'idée païenne de dieu des personnes, comme on parle de dieux des océans, ou de la forêt, ou du feu, ou des tempêtes ou des vents, ou d'autres réalités dynamiques dont on observe les effets comme ceux de forces de la nature. Car comme on l'a vu d'entrée de jeu, la question du soi ne se pose pas seulement au niveau des activités inconscientes de nos cellules, mais, après l'acquisition du langage, d'un «qui » ou d'un «quoi » capable de dire «je». C'est d'ailleurs dans la tension entre ces deux termes, qui ou quoi, que se joue en permanence la réalité de l'individu humain, soit comme personne, soit comme chose. Et quand nous essayons de cerner ce qui se dit ainsi, quand nous disons, en hébreu, ani («je»), nous ne pouvons pas ne pas être arrêtés par une expression curieuse, qui revient très souvent dans la Bible, telle que pour la comprendre, nous devons concevoir cette notion de dieu de la personne.

Cette expression est en hébreu ani («je»), suivi du tétragramme YHVH (Gn 28,13; Nb 28,13; etc.). Elle est traduite en général par «Je suis Dieu », "Je suis l'Éternel », ou «Je suis le Seigneur », suivant ce que l'on choisit pour traduire le tétragramme. Mais tout cela est bien énigmatique. Car, que peut bien vouloir dire, pour un monothéiste bon teint, ce discours de Dieu qui se termine en disant «Je suis Dieu»? Ou bien d'emblée c'est évident, dès que le discours commence par: "Dieu parle...», «Dieu dit...", et cela n'a pas besoin d'être répété en conclusion, ou bien ce n'est pas évident, et le fait de le redire à la fin ne rend pas l'origine divine du discours plus claire pour autant.

En réalité, il existe une autre lecture possible de cette expression, qui utilise le fait que le verbe être, en hébreu, n'est que sous-entendu. Il en résulte que, au lieu de «Je suis [le tétragramme] ", on peut lire "Je est [le tétragramme] ", ou plus précisément «Un “je” [est] [le tétragramme]». Et comme celui-ci est en fait une combinaison des lettres du verbe être dans ses trois temps, une lecture littérale de cette expression pourrait être rendue par: «Un “je” [est] était-est-sera». Alors, au lieu du «Je suis 
Dieu » ou même du "Je suis l'être ", expression d'une tautologie immobile, cette formule peut vouloir nous dire de façon schématique: "Un “je” est être en devenir, que désigne le verbe être aux trois temps. " En cela, nous apprenons quelque chose de plus sur le principal personnage de la Bible, que désigne précisément ce nom, à savoir, que c'est à travers l'expérience du «je» humain, du ani, que s'expérience l'être en devenir.

Autrement dit, il y a là un retournement où l'expérience personnelle humaine est perçue comme ouverture sur une divinité. Car l'expérience d'une existence personnelle, que font les humains quand ils pensent et disent «je», garde toute sa force malgré son caractère problématique. Comme expérience d'une dynamique naturelle, elle peut être perçue comme celle que font les personnes humaines de leurs dieux respectifs, ou anges tutélaires, ou anges gardiens, ou encore "génies » ou dieux particuliers que les anciens Romains attachaient à chaque individu — autant de façons différentes de se percevoir soi-même comme sujet d'une histoire ou d'un destin. L'unification de ces expériences dans l'abstraction d'un "dieu de la personne », ou des personnes, que désignerait l'était-est-sera du tétragramme, exprimerait la possibilité de la réunion des personnes elles-mêmes en une personne humaine collective, ou au moins l'aspiration à une telle réunion.

\subsection{Le paradoxe $d u$ «je»}

Tout cela devient beaucoup plus clair quand on quitte le domaine supposé de la théologie pour celui de la psychologie. Quand on comprend que l'enjeu de ces discours sur les dieux personnels n'est pas l'existence du Dieu de la théologie et de la croyance en des dogmes et articles de foi, mais l'existence des personnes humaines elles-mêmes. Alors, le mot le plus universel pour désigner la divinité de chacun est justement le mot «je », par lequel, dans toutes les langues, celui ou celle qui parle se désigne comme sujet singulier, première personne du singulier. La nature humaine, faite de perceptions de son propre corps, de mémoire, d'imagination et de relation langagière à autrui - humain et non humain —, est telle que chacun se sent coïncider avec la conscience de sa propre existence personnelle. Chacun se perçoit différent de ce qui n'est pas cette conscience, bien que son existence en dépende pourtant, dans l'espace et dans la durée. C'est cette conscience qui lui fait dire «je», notamment quand il se souvient et qu'il fait des projets. Mais ce «je» n'est localisable ni dans l'espace ni dans le temps, sinon de façon triviale dans le corps qui 
parle: le «je» s'évanouit comme un fantôme, un néant sans existence concrète, dès que l'on s'arrête et qu'on essaie de le dénoter.

C'est ce que soulignent certaines interprétations kabbalistes qui s'arrêtent sur le fait que le mot ani («je») est fait des mêmes lettres que le mot aïn («néant»). Pour cette tradition, l'univers est décrit comme un enchaînement infini de mondes, chacun constitué de dix catégories ou sphères, tels que la dixième de l'un est la première du suivant. Et le mot ani désigne la dixième catégorie, tandis que aïn désigne la première. Du fait de cet enchaînement des dizaines l'une après l'autre, c'est la même catégorie du zéro, néant qui n'est pourtant pas rien, qui s'exprime et s'accomplit ensuite à la fin de la dizaine. C'est ainsi une même réalité qui peut être vue soit comme zéro initial, néant ouvert sur le possible, soit comme terminaison d'une entité accomplie qui se nomme «je».

La différence - et la tension entre le «je », comme moi, ego refermé sur soi, et le «je», point d'ouverture sur un infini qui le traverse de part en part - , est exprimée dans une formule ramassée attribuée à Hillel dans le Pirkei Avot ou Traité des Principes: "Im eïn ani li, mi li? Ve-im ani le'atsmi ma ani?» ("Si je [ne suis] pas pour moi, qui [sera] pour moi ? Et si je [suis] pour moi-même, que suis-je ${ }^{1}$ ?») (Pirkei Avot 1,14). Le sens simple est à peu près celui-ci: «Si je ne m'occupe pas de moi de mon salut, notamment par mes œuvres — qui le fera pour moi ? Mais si je suis réduit à moi-même et à mes propres forces, que suis-je? " Mais cette phrase peut être aussi lue d'une autre façon: "Si le "je" est un néant pour moi, alors un qui est pour moi (c'est-à-dire, un questionnement sur le mode personnel, "qui ? ", est pour moi). Mais si le "je" est pour moimême, refermé sur moi, alors "je” est un quoi (une chose).»

On entend là un écho de ces affirmations volontairement paradoxales, sur le mode des kōan bouddhistes, qui font éclater en apparence les principes d'identité et de non contradiction, comme cette affirmation de Yumen Wenyan, maître zen au IX ${ }^{e}$ siècle: "Je suis je parce que je ne suis pas je, et je ne suis pas je, donc je suis $\mathrm{je}^{2} »$.

1. Il n'est pas inintéressant de rappeler que le même Hillel est l'auteur d'une transformation négative du fameux précepte biblique: «Tu aimeras ton prochain comme toi même» (Le 19,18) en un précepte talmudique: "Ce que tu détesterais que l'on te fasse, ne le fais pas à autrui ». Cette injonction est censée résumer toute la Torah, quand il la complète par «Vas et étudie; le reste est interprétation» (Talmud de Babylone, Shabbat, p. 31a).

2. N.D.L.R. Voir l'article de Victor Sōgen Hori dans ce numéro. 


\subsection{Le "je» selon un modèle d'intentionnalité physique}

Dans le contexte moniste que j'annonçais en commençant se pose évidemment la question : comment cette capacité de dire "je» avec les connotations que nous avons brièvement entrevues, peut-elle être produite par, ou associée à, la structure du corps humain et notamment de son cerveau. Sans pouvoir répondre de façon exhaustive à cette question, car nous ne pouvons l'envisager qu'à un niveau tout à fait différent de celui où nous nous sommes situés jusqu'à maintenant, je voudrais seulement mentionner à ce propos un modèle qu'on pourrait qualifier d'intentionnalité physique, c'est-à-dire un modèle de réseaux d'automates simulant des neurones, chacun évidemment non intentionnels, et fonctionnant de façon purement mécanique, tels que leur activité globale fait pourtant émerger des projets nouveaux, non programmés, qui ressemblent à des décisions d'actions volontaires. Celles-ci sont suivies (ou non) de leur exécution et le modèle simule le comportement de sujets conscients, dont des états mentaux singuliers dits intentionnels semblent être la cause d'activités cérébrales et de mouvements du corps qui s'en suivraient.

Ces réseaux simulent des comportements volontaires encore primitifs, tels que nous pouvons les observer chez des grands singes capables d'utiliser des objets comme de nouveaux outils. Vous connaissez le film 2001: l'odyssée de l'espace, de Stanley Kubric, où l'on voit de grands singes jouer avec des ossements jusqu'à ce que l'un d'eux donne un coup à un autre à l'aide d'un de ces os. Il découvre alors que ces ossements peuvent être utilisés comme des armes. Il répète ensuite les mouvements qu'il avait effectués la première fois par hasard, mais cette fois-ci dans le but de donner un coup. Une séquence causale de mouvements est transformée en procédure dirigée vers un but, comme si le projet de donner des coups avec un bâton avait émergé de cette transformation.

Cette forme primitive, mais non triviale, d'intentionnalité peut être simulée sur ordinateur par un réseau auto-organisateur jumelé à une mémoire associative. Très schématiquement, le modèle fonctionne de la façon suivante: une séquence causale d'états du réseau qui a produit la première fois un certain effet (par exemple donner un coup) est stockée en mémoire. Il peut arriver ensuite que le dernier état de cette séquence correspondant à cet effet soit rappelé à l'occasion d'autres événements, d'autres rencontres, par un mécanisme de mémoire associative. Alors, ce qui est rappelé et répété, n'est pas seulement ce dernier état de la 
séquence, mais toute la séquence qui avait produit cet effet la première fois. Comme dans une action volontaire, il existe une inversion apparente du temps où c'est le dernier état — ou un état voisin qui lui est associé - qui est la cause de la répétition de la séquence qui l'avait produite. Autrement dit, une séquence causale est transformée automatiquement en procédure apparemment dirigée vers un but.

Ce que suggère ce modèle d'émergence de projet est que la conscience de soi et des choses, bien qu'elle semble parfois dirigée vers le futur, est en fait une mémoire, dans ce processus de construction de procédures, un rappel d'états qui se sont trouvés être les plus fréquents à cause des propriétés de l'environnement et du réseau à la fois. Un pas de plus vers une intentionnalité humaine impliquerait un autre niveau de mémorisation. Cela permettrait d'étendre le modèle de construction de projets à notre expérience d'une capacité générale de fabriquer des projets, indépendante de tel ou tel projet spécifique. Autrement dit, cela permettrait de concevoir un modèle mécanique de notre conscience comme conscience d'intentionnalité, bien que ce soit encore au sens restreint et limité que nous avons envisagé.

L'idée que la conscience est fondamentalement mémoire d'événements passés, non dirigée ou orientée vers le futur, ne veut pas dire qu'aucune nouveauté n'est à attendre du futur. Mais la nouveauté est d'abord inconsciente $^{3}$. Bien évidemment, avant de survenir, le nouveau n'est pas connu comme tel, n'est pas conscient. Quand il survient, de façon non planifiée et inattendue, et donc, au moins en apparence, aléatoire sinon, ce ne serait pas du nouveau —, la conscience s'en empare après coup. La conscience du nouveau est ainsi le résultat d'une transformation de l'indétermination et du hasard en structure et signification par la dynamique d'une auto-organisation mémorisée.

Ce serait l'une des raisons pour lesquelles nous concevons notre soi de façon confuse, quand nous voulons y voir une origine absolue, douée de libre arbitre en ce qu'il serait capable, par sa volonté consciente, de déclencher des séries causales à partir de rien, sans cause autre que des causes finales, c'est-à-dire des projets qu'il se donnerait à lui-même de façon non déterminée.

3. Dans une réflexion sur le caractère relatif du temps, défini par la dynamique de différents systèmes qui produisent leur propre temps, nous avons suggéré de remplacer «passé» et «futur» par «connu» et «inconnu» (voir Atlan 1982, 24-35). 
À ce propos, il est intéressant de rappeler les résultats obtenus par Benjamin Libet qui mesurait le temps écoulé entre l'activité électrique du cerveau associée à une décision consciente de faire un mouvement volontaire, et l'activité électrique correspondant à l'initiation du mouvement. De façon apparemment paradoxale, il trouvait de manière consistante que l'initiation du mouvement précédait d'environ 300 microsecondes, et non suivait, la conscience d'avoir décidé d'effectuer le mouvement. Ce résultat apparemment paradoxal et heurtant le sens commun, serait le produit d'une antédatation systématique des événements cérébraux conscients. Il s'agirait là d'une espèce d'illusion temporelle construite par le cerveau, analogue aux illusions spatiales d'optique bien connues où le cerveau construit des images différentes de ce qu'elles sont quand on les décrit objectivement. Cette observation, répétée depuis, a donné lieu à beaucoup de contestations et d'incompréhensions, qui s'atténuent considérablement si l'on comprend, comme le dit Spinoza et comme le suggère aussi notre modèle d'intentionnalité et d'émergence des projets, que "l'entendement et la volonté sont une seule et même chose (Spinoza 1990, 49), c'est-à-dire la décision volontaire et l'affirmation d'un état de choses sont une seule et même chose vue dans deux perspectives temporelles différentes: apparemment orientée vers l'avenir dans un cas, répétition du passé dans l'autre.

Ces modèles d'auto-organisation mémorisée nous montrent comment, contrairement à la conception idéalisée de la conscience comme visée vers, orientée vers un futur inexistant ou simplement vers ce qui est hors d'elle, la conscience serait fondamentalement mémoire d'états et de processus passés. Par contre, l'intentionnalité créatrice, inventrice de projets et donneuse de significations, serait le propre de phénomènes auto-organisateurs d'abord inconscients, produits par l'émergence de structures et de fonctions nouvelles, comme dans nos réseaux d'automates. Ces structures et fonctions nouvelles apparaîtraient évidemment comme telles dans une activité secondaire, lorsque la conscience mémoire observerait cette émergence et la mémoriserait à son tour. Comme nous l'avions suggéré autrefois, notre conscience volontaire serait le résultat de mémorisation de phénomènes auto-organisateurs, tandis que ceux-ci produisent, de façon inconsciente, la nouveauté de l'avenir. Ce serait cette association d'un vouloir inconscient à une conscience-mémoire qui instituerait l'unité temporelle fragile d'un soi. Une conscience intentionnelle 
ou une intention consciente apparaît alors non comme un phénomène premier, fondateur, mais comme secondaire, produite par cette association (Atlan 1979, chap. 5; pour un exposé plus détaillé des notions présentées ici, voir Atlan 2003, chap. 5, 7.).

\section{Post-scriptum \\ Dialogue}

Paul Dumouchel

Département de philosophie Université du Québec à Montréal Graduate School of CoreEthics and Frontier Sciences Ritsumeikan University

Une des questions que soulève cet article, me semble-t-il, est celle de savoir quel rôle joue la science dans ce dialogue. Qu'apporte la science à cette discussion au sujet du soi, par rapport au droit ou à la pensée religieuse, hébraïque en l'occurrence? Pourquoi avons-nous besoin de la science comme interlocuteur? Que vaut la connaissance scientifique pour connaître le soi ?

Cette dernière question nous incite à revenir en arrière, car elle renvoie à une autre question, non formulée celle-là, et à laquelle elle fournit une réponse: quel est ce dialogue? Et elle répond: son but est de connaître le soi. Mais en va-t-il bien ainsi ? Je ne crois pas. Le but de ce dialogue n'est pas la connaissance. Cette discussion entre les neurosciences, la philosophie et la théologie existe, me semble-t-il, parce qu'elle est inévitable. Elle ne vise pas un but particulier; cette discussion est tout simplement ce que nous sommes. Elle reflète que nous sommes inévitablement partagés entre trois sources de savoir pour guider notre action. Premièrement, les sciences, car elles constituent à l'heure actuelle la meilleure connaissance que nous possédions au sujet du monde. Deuxièmement, une réflexion autonome, car c'est de notre propre action dont il s'agit. Nous en sommes les parties prenantes et c'est nous qui en souffrirons les 
conséquences. Il n'y a pas lieu de laisser à d'autres le soin de décider. Enfin troisièmement, une tradition à laquelle nous ne pouvons entièrement renoncer sous peine de cesser d'être nous-mêmes, mais dont nous ne savons pas toujours très bien comment elle peut s'accommoder et de la science et de la réflexion autonome. Car, comme le dit si bien Henri Atlan, ce dialogue est "plus difficile aujourd'hui qu'autrefois, puisque l'illusion de l'unité toute faite entre vérité et justice, entre le vrai et le bien, a été dissipée.» ( $\mathbb{S} 2.4$. de cet article) Or, ces différentes traditions religieuses auxquelles nous nous rattachons nous ont précisément été léguées comme le lieu de cette unité, mais elles ne nous apparaissent souvent aujourd'hui plus que comme un savoir périmé et un obstacle à l'autonomie de la réflexion.

Ce dialogue est inévitable, car il prend place tous les jours, dans les hôpitaux, les cliniques, les laboratoires, mais aussi dans les ministères, dans les bureaux des entreprises de biotechnologies, dans les journaux et à la télévision; bref, partout où des décisions se prennent et où l'opinion se forme. Il s'agit donc, comme le disait Hannah Arendt de "penser ce que nous faisons ». Or, la recommandation première qui nous est donnée par Atlan est, me semble-t-il, qu'il nous faut « renoncer à l'idée de définir la personne humaine comme une réalité dans l'ordre des faits, si ces faits ne doivent être instruits que par les sciences de la nature - biologie et psychologie comprises - et qu'au contraire, il faut accepter l'idée qu'il s'agit là d'une réalité de droit.» ( $\mathbb{S} 2.1$. de cet article) D'où la question par laquelle je commençais ces quelques remarques: quel est le rôle de la science dans ce dialogue? Quelle place peut avoir la science une fois que nous avons abandonné la possibilité de définir la personne humaine dans l'ordre des faits? La réponse à cette dernière question n'est pas difficile à trouver: la place que la science occupe de droit et de fait ici tient à ce qu'elle est le moyen par excellence de notre action. Car la science n'est pas seulement connaissance; elle est aussi savoir-faire. C'est ce «faire» qui plus que tout pose problème.

Par contre, le rôle que la science joue dans ce texte est différent de la place qu'elle y occupe. Ce rôle est paradoxal dans la mesure où il consiste souvent à s'effacer en laissant à d'autres voix l'occasion de se faire entendre. Je veux dire par là que les références scientifiques du texte d'Henri Atlan ne viennent pas saper l'autorité d'autres formes de savoirs, mais au contraire interviennent le plus souvent pour rectifier une image trop simple du déterminisme scientifique et de ses conséquences. À 
d'autres moments, elles sont là pour enrichir le dialogue, mais toujours elles ne constituent que des éléments de réponse parmi d'autres. La science n'est pas ici, pas plus que le droit ou la pensée religieuse, le dépositaire de l'Autorité.

Ceci dit, définir la personne humaine dans l'ordre du droit ne va pas sans difficultés. En effet, le droit est culturellement relatif. Il est sujet aux accidents du temps et du lieu. Comment la personne, une invention du droit romain, pourrait-elle soutenir l'édifice universel des droits de l'homme? Pourquoi ces deux principes - le partage entre, d'une part, les humains et, d'autre part, les choses et les autres vivants premièrement; l'indivisibilité du corps et de l'esprit deuxièmement - doivent-ils nous tenir lieu de règle? La réponse qu'apporte Henri Atlan à cette question à partir de sa tradition est subtile et intéressante pour plusieurs raisons.

La tradition hébraïque, tout comme le droit romain, définit la personne socialement. La personne humaine se découvre dans la rencontre de deux individus de sexes différents et se fonde sur la reconnaissance de la forme partagée du corps humain. Dès lors, on retrouve les deux mêmes caractéristiques que dans le droit romain: un statut qui sépare l'être humain des choses et des autres vivants, et le fait que la personne ne peut pas exister comme une entité abstraite indépendante du corps, dont la forme ici est le principe immédiat de reconnaissance. Cette forme que nous reconnaissons tous spontanément, par un geste qui abolit les différences de cultures et de savoir, peut, propose Atlan, servir encore aujourd'hui de guide et de repère pour des questions difficiles concernant l'avortement ou l'expérimentation sur des animaux ou des cellules humaines.

Aussi séduisante que soit cette proposition un moment de réflexion suffit pour se convaincre qu'elle n'est pas dépourvue de difficultés. En effet, combien différente doit être la forme de l'autre afin de ne plus être la forme d'un corps humain? Quel est le point de difformité qui détermine qu'un nouveau-né n'est pas un enfant? Il n'y a pas, je crois, de réponses définitives à ces questions. Selon les cultures, les époques, les croyances et les préjugés, on y apportera des réponses différentes qui nous paraîtront selon le cas plus ou moins satisfaisantes. À cette difficulté, Henri Atlan suggère, semble-t-il, le remède suivant: «En fait, c'est le sens de notre sensibilité immédiate qu'il s'agit finalement de retrouver et d'assumer, plutôt que de chercher une illusoire définition essentialiste de la nature de la personne humaine»( $\mathbb{S} 2.3$ de cet article). Je suis 
d'accord avec la seconde partie de cette phrase, mais je crois que nous avons d'excellentes raisons théoriques (Dumouchel 2004) et pratiques, ou, si on préfère, empiriques, de se méfier des appels "au sens de notre sensibilité ». Les arrêts de la sensibilité sont tout aussi incertains que ceux des préjugés et des croyances multiples. Ils sont cependant beaucoup plus imprévisibles et inaptes au dialogue, car la sensibilité constitue toujours en définitive son ultime critère, son indépassable pierre de touche. Je veux dire par là que le danger de l'appel à la sensibilité immédiate comme critère est que celle-ci menace toujours de se réduire à l'autorité de la première personne, une autorité indépassable, que nul ne peut remettre en cause et qui, pour cette raison même, met un terme au dialogue.

Il convient plutôt, je pense, de reconnaître que l'incapacité de la reconnaissance de la forme du corps humain à répondre sans ambiguité à ces questions de limites n'est pas dirimante, car elle renvoie à une incertitude qui est, en un sens, indépassable. Cette incertitude constitue un aspect de la condition humaine que nous ne pouvons récuser. Tout dans le texte d'Atlan, me semble-t-il, conduit à cette conclusion. En fait, la reconnaissance de la forme du corps humain entre l'homme et la femme contient déjà une différence. Atlan de dire:

Autrement dit, la personne humaine serait instituée, non pas dans un individu - c'est pour cette raison qu'il n'y a pas de mot unique pour la désigner - , mais dans la rencontre et la reconnaissance par le regard d'un autre. Et pas de n'importe quel autre: pas l'animal, trop différent, et pas l'autre du même sexe, trop semblable, mais l'autre du sexe différent. ( $\mathbb{S} 2.3$. de cet article)

La personne humaine surgit de la reconnaissance d'une différence dans l'identité. Un autre qui n'est ni trop semblable, ni trop différent. Notre capacité à nous identifier plus ou moins à différents animaux est aussi ce qui guide, ou ce qui pourrait guider, selon Atlan, notre jugement de ce qui est plus ou moins acceptable en ce qui a trait à l'expérimentation sur des animaux ou des cellules humaines. Or, cette capacité, même si nous la qualifions souvent de "spontanée » et «immédiate », n'est pas donnée d'emblée. Elle est au contraire, comme le soi, le résultat d'une histoire et d'un constant dialogue. Cela ne devrait ni nous surprendre ni nous inquiéter, car dire que toute définition essentialiste de la personne est illusoire revient à dire qu'il n'y a pas de liste de propriétés qui permette de dire à coup sûr et de façon définitive qui est une personne. C'est au dialogue de le dire. Cependant, comme un dialogue est tout le 
contraire du monologue dogmatique des experts, son enjeu n'est pas de fournir une réponse qui va mettre un terme à la discussion. Il ne peut être que de proposer des réponses provisoires toujours susceptibles d'être remises en question et remplacées. En ce sens, ce dialogue ressemble assez à une certaine image de la science appréhendée comme faillible, où nos meilleures théories peuvent à tout moment être trouvées inadéquates et rejetées. Il s'en distingue par contre en ceci que, à tort ou à raison, dans les sciences, nous sommes persuadés que les nouvelles théories sont supérieures à celles qu'elles remplacent. Il n'en va pas de même ici: l'incertitude qui nous tenaille n'est pas compensée par le savoir que nos décisions présentes l'emportent sur celles d'hier, car très souvent la difficulté vient de ce que les solutions passées ne sont plus disponibles. Elles ne font plus partie de l'ensemble de choix qui nous est offert. En conséquence, il me semble que ce dialogue ne peut pas avoir d'autre but que le dialogue lui-même. Non pas au sens où il serait incapable de donner réponse ou de guider l'action, mais au sens où toute réponse est provisoire et toute action faillible. Dès lors, l'intérêt de ce critère qu'est la reconnaissance de la forme humaine est justement qu'il ne correspond pas à une évidence irrécusable et qu'il permet, de ce fait, la reprise et la poursuite d'un dialogue dont nul n'est exclus.

\section{Références}

Arendt, H. (1961), [anglais 1958] Condition de l'homme moderne / trad. par G. Fradier, Paris, Calmann-Lévy, 1961.

Atlan, H. (1979), Entre le cristal et la fumée, Paris, Seuil.

- (1982), "Temps biologique et auto-organisation", dans H. Atlan, Temps de la vie et temps vécu, Paris, CNRS, p. 24-35.

— (2003), Les étincelles de hasard, t. II : Athéisme de l'Écriture, Paris, Seuil.

Dumouchel, P. (2004), "Y a-t-il des sentiments moraux? », Dialogue, 43/3, p. 471-489.

SPINOZA, B. (1990), [latin 1663-1677] Éthique / introduction, traduction, notes, commentaires et index par R. Misrahi, Paris, Presses universitaires de France (Philosophie d'aujourd'hui). 


\section{Résumé}

La définition factuelle du soi conscient semble échapper à la science. Une manière non essentialiste d'appréhender le soi pourrait être de revisiter les notions juridiques de «personne» et de «je », considérés ici du point de vue de la tradition juive (Bible, Talmud, Kabbale), éventuellement complétée et éclairée par Spinoza, et dans une perspective concrète collective: "de quoi parlons-nous quand nous parlons du soi?» D'une part, Genèse 1-2 met en intrigue la "personne» non pas d'abord comme individualité, mais comme devenir en jeu dans la rencontre homme-femme - ce qui nous permet de considérer comme appartenant à l'ordre des "personnes » celui ou celle chez qui on reconnaît la forme (tsélem) de l'espèce humaine. D'autre part, la relecture de l'énoncé biblique "Je YHWH» $(\mathrm{Gn} 28,13)$ sous l'angle de la symbolique mythique du dieu des personnes, permet d'appréhender le soi comme acte de langage paradoxal, à situer entre autoaffirmation et néant. Or, il est possible de confronter cette capacité de dire «je " à un modèle informatique d'intentionnalité physique qui décrit le fonctionnement des neurones comme un réseau d'automates ou auto-organisation mémorisée. Par là, le dialogue entre sagesse pratique et science, d'entrée de jeu mis entre parenthèse, revient à l'avant-plan.

\section{Abstract}

A fact-based definition of the conscious self seems to be lacking in science. A nonessentialist approach of understanding the self would be to examine two legal concepts, "personhood" and the "I", considered in this work from the point of view of the Jewish tradition (that is the Bible, the Talmud and Kabbalah), eventually completed with Spinozist philosophy, and from a collective pragmatic perspective: "What do we mean when we talk to one another about ourselves and other selves?" On the one hand, the storyline of Genesis 1-2 does not introduce a "person" in its individuality (the name for person does not exist in Hebrew as a substantive), but as a mean of bringing into play male and female. This allows us to consider that any being in which the form of a human body (the tselem) is recognized belongs to the category of "persons". On the other hand, the symbolic, mythical interpretation of the biblical phrase, "I YHWH", repeated again and again, as the god of persons, permits an understanding of the self as a paradoxical speech act, positioned between self-affirmation and nothingness. At the same time, it is possible to confront this capacity to say "I" to a computer model of "physical intentionality". This model describes the actual production of individual projects, as emerging from the activity of a network of neuron-like automata, functioning as a memorized self-organization. This is the way through which the dialogue that has been relegated to the sidelines between practical wisdom and science can return to the forefront.

(C) Revue Théologiques 2004. Tout droit réservé. 